\title{
Long non-coding RNA expression profiling following treatment with resveratrol to improve insulin resistance
}

\author{
LINYI SHU ${ }^{1,2}$, GUANGSEN HOU ${ }^{1,2}$, HANG ZHAO $^{2}$, WENLI HUANG ${ }^{1,2}$, \\ GUANGYAO SONG ${ }^{1,2}$ and HUIJUAN MA ${ }^{1-3}$ \\ ${ }^{1}$ Department of Internal Medicine, Hebei Medical University, Shijiazhuang, Hebei 050017; ${ }^{2}$ Endocrinology Department; \\ ${ }^{3}$ Hebei Key Laboratory of Metabolic Diseases, Hebei General Hospital, Shijiazhuang, Hebei 050051, P.R. China
}

Received November 15, 2019; Accepted May 18, 2020

DOI: $10.3892 / \mathrm{mmr} .2020 .11221$

\begin{abstract}
Resveratrol (RSV) and long non-coding RNAs (lncRNAs) play a role in the treatment of diabetes; however, the mechanism by which resveratrol regulates insulin resistance via lncRNAs is currently unknown. The present study aimed to determine the lncRNA expression level profile in mice following resveratrol treatment to improve insulin resistance using high-throughput sequencing technology. C57BL/6J mice were fed a high-fat diet for 8 weeks to develop an insulin resistance model, followed by treatment with or without RSV for 6 weeks before high-throughput sequencing. Following RSV treatment, 28 and 30 lncRNAs were up- and downregulated, respectively; eight IncRNAs were randomly selected and evaluated using reverse transcription-quantitative PCR, which showed results consistent with the sequencing analysis. Pathway analysis demonstrated that the insulin signaling pathway enrichment score was the highest, and identified two lncRNAs, NONMMUT058999.2 and NONMMUT051901.2, consistent with the protein-encoding genes SOCS3 and G6PC, respectively. Similar expression level patterns were observed for SOCS3 and G6PC, suggesting that RSV improves insulin resistance by modulating lncRNAs. RSV decreased the expression levels of SOCS3, FOXO1, G6PC and PEPCK in mice. The same results were observed following knockdown of NONMMUT058999.2 in cells. The present study provides a new biomarker or intervention target
\end{abstract}

Correspondence to: Professor Guangyao Song, Endocrinology Department, Hebei General Hospital, 348 Heping West Road, Shijiazhuang, Hebei 050051, P.R. China

E-mail: guangyaosong123@163.com

Abbreviations: BP, biological process; CC, cellular component; FOXO1, forkhead box O1; G6PC, glucose-6-phosphatase catalytic subunit; HFD, high-fat diet; MF, molecular function; miRNA, microRNA; PEPCK, phosphoenolpyruvate carboxykinase; SOCS3, suppressor of cytokine signaling 3

Key words: resveratrol, long non-coding RNA, high-throughput sequencing, insulin resistance, diabetes mellitus for RSV in the treatment of diabetes, and a new perspective for understanding the hypoglycemic mechanism of RSV.

\section{Introduction}

Type 2 diabetes mellitus (T2DM) is a metabolic disease with a complex etiology in which patients show a chronically high blood sugar level (1). A previous study demonstrated that the number of adults with diabetes worldwide was $\sim 425,000,000$ in 2015 (1). The incidence rate is predicted to reach $629,000,000$ by 2040 (1). The pathogenesis of T2DM is complex, with it primarily being caused by decreased insulin secretion from pancreatic islet $\beta$ cells following the development of insulin resistance (IR) in target organs and tissues (primarily the liver, muscle and fat) (2). As a key site of glycogen metabolism in the human body, the liver is also the primary organ of gluconeogenesis. It can regulate the insulin-inhibiting and glucagon-stimulated hepatic gluconeogenesis pathway, as well as maintain the body's blood sugar within normal levels. IR can lead to relative or absolute deficiency of insulin; additionally, unrestricted gluconeogenesis leads to prolonged hyperglycemia and eventually diabetes (3). Therefore, decreasing hepatic glucose production and hepatic insulin resistance may be useful for treating T2DM.

Resveratrol (RSV) is a natural polyphenolic compound mainly derived from red grapes, blueberries, mulberries, peanuts and other plants (4). This compound has numerous pharmacological activities, can protect the cardiovascular and nervous systems, and exerts antitumor, anti-inflammatory and immunoregulatory effects (5). In the past decade, studies have demonstrated that RSV can activate insulin signaling pathways to improve the effects of IR and protect pancreatic islet $\beta$ cells $(6,7)$. RSV can also play a beneficial role in improving insulin sensitivity including histone deacetylase 4 and decreasing liver endoplasmic reticulum stress $(8,9)$. Resveratrol promotes the translocation of histone deacetylase 4 from the nucleus to the cytoplasm, and histone deacetylase 4 may be an agonist of resveratrol (9).

Long non-coding RNAs (lncRNAs) are RNA molecules $>200$ nucleotides in length that do not encode proteins. These molecules are involved in regulating proliferation, differentiation and apoptosis (10). A previous study showed 
that IncRNA plays a role in the development of T2DM and can be used as a target for the diagnosis and treatment of diabetes (10). The expression levels of lncRNA H19 were significantly decreased in the skeletal muscle of patients with type 2 diabetes and insulin-resistant animals (11). As both RSV and lncRNAs are involved in the development of T2DM and improve IR, there may be an association between these molecules. Little is currently known about whether IncRNAs are involved in the RSV-mediated effects of improving IR in the liver. The present study analyzed the expression level profile of RSV in the liver of insulin-resistant lncRNAs in mice induced by a high-fat diet (HFD), and investigated the role of RSV in improving IR via IncRNAs. Reverse transcription-quantitative (RT-q) PCR was performed to verify the expression levels of eight lncRNAs, providing a foundation for the prevention and treatment of diabetes.

\section{Materials and methods}

Materials. A total of 36 6-week-old C57BL/6J male mice (21-25 g) were purchased from Beijing Weitong Lihua Experimental Animal Center [license no. SCXK (Beijing) 2016-0006; Beijing, China] and housed in the animal laboratory barrier system of Hebei Provincial People's Hospital (20-25 $\mathrm{C}$, relative humidity 40-60\%, 12-h light/dark cycle and access to food/water provided ad libitum). Following 1 week of acclimation, the mice were divided into control $(\mathrm{CON} ; \mathrm{n}=12)$ and HFD groups $(\mathrm{n}=24)(\mathrm{CON}$ feed D12450J: $3.85 \mathrm{kcal} / \mathrm{g}$; protein, 20; carbohydrate, 70; fat, 10\%; HFD feed D12492: $5.24 \mathrm{kcal} / \mathrm{g}$; protein, 20; carbohydrate, 20; fat, 60\%). The feed was purchased from Beijing Huafukang Biotechnology Co., Ltd. Following 8 weeks of feeding, the rats were injected with an intraperitoneal glucose tolerance test for $12 \mathrm{~h}$ : According to the body weight of mice, $2 \mathrm{~g} / \mathrm{kg}$ glucose saline (50\% glucose injection: $0.9 \%$ sodium chloride solution prepared in a 1:1 ratio) was injected intraperitoneally at 0,15, 30,60 and $120 \mathrm{~min}$. The blood glucose at the corresponding time point was measured, and the degree of insulin resistance and the success of establishing the insulin resistance model were evaluated. The IR model was successfully established from the HFD group according to calculation of the area under the curve. A total of 12 mice in the HFD group continued high-fat feeding, and 12 mice were treated with HFD+RSV. RSV was dissolved in DMSO (both from Sigma-Aldrich; Merck KGaA), diluted with $0.9 \%$ sodium chloride solution, and administered at $100 \mathrm{mg} / \mathrm{kg}$ per day intragastrically (12). Next, the remaining two groups of mice were administered $0.1 \%$ DMSO in $0.9 \%$ sodium chloride solution for intragastric delivery intervention. Following 6 weeks of resveratrol treatment, the mice were fasted for $12 \mathrm{~h}$ overnight. A total of three mice were randomly selected from each group to inject $1.5 \mathrm{IU} / 40 \mathrm{~g}$ of insulin (Sigma-Aldrich; Merck KGaA) intraperitoneally $20 \mathrm{~min}$ before anesthesia. All mice were sacrificed by cervical dislocation. The liver was dissected in all mice, and a small piece of the liver was fixed in $4 \%$ paraformaldehyde $\left(4^{\circ} \mathrm{C}\right.$ for $24 \mathrm{~h}$ ). The remaining tissue was frozen in liquid nitrogen and stored at $-80^{\circ} \mathrm{C}$. These liver tissues can be maintained for at least 6 months. The experiment was supervised and approved by the Ethics Committee of the People's Hospital of Hebei Province (approval no. 201920) and performed in accordance with the Regulations on the Administration of Laboratory Animals.
High-throughput sequencing. A total of four segments were selected from each of the liver samples in the CON group, HFD group and HFD+RSV group for extraction of total RNA using the RNeasy mini kit (Qiagen $\mathrm{GmbH}$ ). The sequencing library was constructed using TruSeq ${ }^{\mathrm{TM}}$ RNA (Illumina, Inc.). The purified cDNA was amplified by removing the ribosomal RNA, replicating the first-strand cDNA, and synthesizing the second-strand cDNA to generate the final cDNA library. This was performed according to the manufacturer's instructions. The insert size was verified using Qubit ${ }^{\circledR} 2.0$ (Thermo Fisher Scientific, Inc.) and Agilent 2100 Bioanalyzer (Agilent Technologies, Inc.), and molar concentrations were calculated and then sequenced using a NovaSeq 6000 (Illumina, Inc.). Library construction and sequencing were performed by Shanghai Sinomics Corporation. Both lncRNA and mRNA were analyzed using databases; lncRNA was evaluated using RefSeq, Ensembls and Genebank, and mRNA was evaluated using Noncode and Ensembls. The high-throughput sequencing results were uploaded to the Gene Expression Omnibus database (accession no. GSE137840) (12).

Expression level analysis of lncRNAs and mRNAs. Fragments of each gene segment were compared using Stringtie (13) software, and the fragments per kilobase of million mapped reads (FPKM) value of each gene was calculated using the trimmed mean of P-value. Differences in gene expression levels between groups were analyzed using edgeR (14) software. The P-value corrected after multiple comparison tests is termed Q-value and these parameters are used to statistically screen differential genes. Additionally, the differential expression level fold-change was calculated according to the FPKM value, and the $\log _{2}$ (fold-change) was calculated for subsequent screening of differential genes (15).

Reversetranscription-quantitative $P C R(R T-q P C R)$. TotalRNA was extracted with TRIzol ${ }^{\circledR}$ reagent (Thermo Fisher Scientific, Inc.) from mice liver tissues, and the RNA concentration and purity were determined using a NanoDrop 2000 (Thermo Fisher Scientific, Inc.). RNA was reverse-transcribed into cDNA using HiScript II Q RT SuperMix for qPCR (RR047A; Takara Bio, Inc.). Amplification was performed using the SYBR $^{\circledR}$ Premix Ex TaqTM II kit (RR820A; Takara Bio, Inc.). PCR was performed using Applied Biosystems 7900 apparatus (Thermo Fisher Scientific, Inc.) at $95^{\circ} \mathrm{C}$ for $10 \mathrm{~min}$, followed by 40 cycles for $15 \mathrm{sec}$ at $95^{\circ} \mathrm{C}, 15 \mathrm{sec}$ at $95^{\circ} \mathrm{C}$ and $15 \mathrm{sec}$ at $60^{\circ} \mathrm{C}$. Gene expression levels were normalized to those of $\beta$-actin using the $2^{-\Delta \Delta \mathrm{Cq}}$ method (16). The primer sequences are listed in Table I.

Western blotting analysis. Protein was extracted with $1.4 \%$ SDS and concentration was determined using a BCA kit (Thermo Fisher Scientific, Inc.). SDS-PAGE gels (Beijing Solarbio Technology Co., Ltd.) were prepared at different concentrations $(8,10$ and $12 \%)$. The amount of protein loaded per lane was $\sim 30-50 \mu \mathrm{g}$. The protein was transferred to a PVDF membrane using electrophoresis and then blocked with $5 \%$ skimmed milk for $3 \mathrm{~h}$ at $4^{\circ} \mathrm{C}$. After diluting the primary antibodies (20\% Tween TBST dissolved in 5\% skimmed milk, $20 \%$ Tween TBST dissolved in 5\% BSA for phosphorylated proteins), the membrane was incubated with the antibody at 
Table I. Reverse transcription-quantitative PCR primers.

Gene

$\beta$-actin

NONMMUT119418.1

NONMMUT058999.2

NONMMUT068763.2

NONMMUT051901.2

NONMMUT010559.2

NONMMUT059852.2

NONMMUT027048.2

NONMMUT001352.2

FOXO1

G6PC

SOCS3

PEPCK
Forward primer, 5'-3'

Reverse primer, 5'-3'

GGGATGTTTGCTCCAACCAA

CTACCCTGTTCCTGTGCATTCC

GGCCGCAGGTAAGGGAGATT

GACCCGGCTTCATTCATCTTTC

CACCCAGCTTATTCCAGTCCTCT

TGGAGGCTTTACCAGATGTGAG

ACCTACACGAAGCCATCCAAAA

CATAAATGGAGGTTAGTAGGTGGC

ATGGCTGCATCATATCAGTTGG

GATTTTCCGCTCTTGCCTCC

TAGGCTGAGGAGGAGAAAACTG

GAATCCCTCAACTCTCTGCCTA

CTGGCTGATTCTCTGTTTCAGG $4^{\circ} \mathrm{C}$ overnight, followed by washing three times $(10 \mathrm{~min}$ each time) with TBST (20\% Tween), incubation with the secondary antibody for $\sim 50 \mathrm{~min}$ at room temperature, and washing three times (10 min each time). Finally, the protein band was observed with a gel imager (GDS8000; UVP Products) and the grey value was read using the ImageJ 1.8.0 (National Institutes of Health) software. Standardization was performed by incubating the antibody with $\beta$-actin. The primary antibodies were as follows: Anti- $\beta$-actin (mouse; 1:1,000; cat. no. 3700S; Cell Signaling Technology, Inc.); anti-forkhead box O1 (FOXO1; 1:1,000; cat. no. 18592-1-AP); anti-glucose-6-phosphatase catalytic subunit (G6PC; 1:2,000; cat. no. 22169-1-AP); anti-suppressor of cytokine signaling 3 (SOCS3; 1:1,000; cat. no. 14025-1-AP) (all rabbit antibodies from ProteinTech Group, Inc.); rabbit anti-phosphoenolpyruvate carboxykinase (PEPCK; 1:1,000; cat. no. 702748; Thermo Fisher Scientific, Inc.); horseradish peroxidase (HRP)-labelled goat anti-rabbit IgG antibody and goat anti-mouse $\operatorname{IgG}$ antibody $(1: 8,000$; cat. no. L3012-2; and 1:3,000, cat. no. L3032-2 respectively; both from Signalway Antibody LLC).

Establishment of cell model and analysis. Mouse liver cancer cells (Hepa) were purchased from the cell bank of the Chinese Academy of Sciences (Beijing, China). Hepa cells were cultured in DMEM (Gibco; Thermo Fisher Scientific, Inc.) supplemented with $10 \%$ fetal bovine serum (HyClone; GE Healthcare Life Sciences), 1\% non-essential amino acids (Gibco; Thermo Fisher Scientific, Inc.) and 1\% streptomycin (HyClone; GE Healthcare Life Sciences). In order to establish the IR model, the cells were transferred to DMEM with $0.25 \mathrm{mmol} \mathrm{L-1}$ palmitic acid (PA) upon reaching $\sim 80 \%$ confluency (17). The cells were transfected in an incubator at $37^{\circ} \mathrm{C}$ for $24 \mathrm{~h}$. The transfection complex 200 Opti-MEM (Gibco; Thermo Fisher Scientific, Inc.), 5 RNA oligo (Suzhou Pharmaceutical Technology Co., Ltd) stock solution and $10 \mu \mathrm{l}$ siRNA-mate transfection reagent (Suzhou Pharmaceutical Technology Co., Ltd) was added to the medium at a final concentration of $50 \mathrm{nM}$ siRNA. The cells were divided into two groups: siRNA-NONMMUT058999.2 [forward primer, 5'-3'-GGCCUGACUUAAGAGUUAAGU; reverse primer, 5'-3'-UUAACUCUUAAGUCAGGCCAG; Suzhou Pharmaceutical Technology Co., Ltd. (negative control, NC) and siRNA-NONMMUT058999.2 knockdown (knockdown)]. The groups were treated with $200 \mu \mathrm{l}$ of transfection complex in $1.8 \mathrm{ml}$ of medium per well, and the final siRNA concentration was $\sim 50 \mathrm{nM}$. The cells were cultured at $37^{\circ} \mathrm{C}$ for $24 \mathrm{~h}$. Then RNA was extracted and the success of NONMMUT058999.2 knockdown verified by RT-qPCR. After knockdown of NONMMUT058999.2, Hepa cells were seeded in 6-well plates, $24 \mathrm{~h}$ after transfection. PA and RSV $30 \mu \mathrm{M}$ were added to the corresponding groups (18). Proteins were extracted for western blotting $40 \mathrm{~min}$ following insulin stimulation.

lncRNA-miRNA-mRNA co-expression network. Competing endogenous (ce)RNA analysis is based on the expression value of genes. Using regression model analysis and seed sequence matching methods, a regulatory network of microRNA sponge adsorption was established to find the core ceRNAs $(19,20)$.

Statistical analysis. All data were processed using SPSS 21.0 software (SPSS, Inc.) and the results were expressed as the mean \pm standard deviation (SD). Multiple groups were compared using one-way analysis of variance (ANOVA) followed by least significant difference or Tamhane's test. $\mathrm{P}<0.05$ was considered to indicate a statistically significant difference. For lncRNAs and mRNAs, significant levels of change were expressed as a $\mathrm{Q}$ value $<0.05$ and $\log _{2}$ (fold-change) $\geq 1$.

\section{Results}

Expression level profiles of lncRNAs and mRNAs. High-throughput sequencing was performed to detect the expression levels of IncRNAs and mRNAs in the liver samples from each group. A total of 51,024 lncRNAs and 31,055 mRNAs were detected. Additionally, 58 differences were detected between the HFD + RSV and HFD groups. The expressed lncRNAs (28 upregulated and 30 downregulated) 
Table II. Expression level patterns of lncRNAs in HFD + RSV vs. HFD mice.

\begin{tabular}{|c|c|c|c|}
\hline Gene & $\log _{2}$, fold-change & Q-value & Regulation, HFD + RSV vs. HFD \\
\hline ENSMUST00000180982 & 8.66426 & $2.38 \times 10^{-5}$ & Up \\
\hline NONMMUT034345.2 & 6.58843 & $5.82 \times 10^{-7}$ & Up \\
\hline NONMMUT053361.2 & 6.47468 & $1.03 \times 10^{-2}$ & Up \\
\hline NONMMUT057779.2 & 6.15313 & $1.03 \times 10^{-2}$ & $\mathrm{Up}$ \\
\hline NONMMUT069202.2 & 6.00386 & $4.06 \times 10^{-2}$ & Up \\
\hline NONMMUT147866.1 & 5.91096 & $4.01 \times 10^{-3}$ & Up \\
\hline NONMMUT154084.1 & 5.46016 & $1.55 \times 10^{-3}$ & Up \\
\hline NONMMUT010559.2 & 5.39012 & $6.08 \times 10^{-21}$ & Up \\
\hline NONMMUT059852.2 & 5.13583 & $1.12 \times 10^{-12}$ & Up \\
\hline NONMMUT039378.2 & 4.67745 & $1.66 \times 10^{-2}$ & Up \\
\hline ENSMUST00000193029 & 4.66360 & $3.98 \times 10^{-7}$ & Up \\
\hline NONMMUT050350.2 & 3.97316 & $2.56 \times 10^{-2}$ & Up \\
\hline NONMMUT057244.2 & 3.90189 & $1.55 \times 10^{-3}$ & Up \\
\hline NONMMUT027048.2 & 3.85579 & $2.97 \times 10^{-2}$ & Up \\
\hline NONMMUT001352.2 & 3.81448 & $6.36 \times 10^{-7}$ & Up \\
\hline NONMMUT062675.2 & 3.67421 & $4.45 \times 10^{-3}$ & Up \\
\hline NONMMUT069358.2 & 3.40734 & $1.01 \times 10^{-2}$ & Up \\
\hline NONMMUT077969.1 & 3.31443 & $3.29 \times 10^{-2}$ & Up \\
\hline NONMMUT035436.2 & 3.25810 & $2.83 \times 10^{-2}$ & Up \\
\hline NONMMUT001470.2 & 3.22941 & $2.31 \times 10^{-4}$ & Up \\
\hline NONMMUT017329.2 & -1.52654 & $2.83 \times 10^{-2}$ & Down \\
\hline NONMMUT153837.1 & -1.90270 & $2.29 \times 10^{-2}$ & Down \\
\hline ENSMUST00000156612 & -2.00163 & $2.15 \times 10^{-2}$ & Down \\
\hline NONMMUT044184.2 & -2.01973 & $4.38 \times 10^{-2}$ & Down \\
\hline ENSMUST00000194058 & -2.15526 & $4.01 \times 10^{-3}$ & Down \\
\hline MSTRG.5260.1 & -2.40263 & $4.01 \times 10^{-3}$ & Down \\
\hline NONMMUT010788.2 & -2.48785 & $3.09 \times 10^{-2}$ & Down \\
\hline NONMMUT119418.1 & -2.57853 & $3.51 \times 10^{-2}$ & Down \\
\hline NONMMUT059480.2 & -2.63681 & $2.13 \times 10^{-2}$ & Down \\
\hline NONMMUT047505.2 & -2.64522 & $1.53 \times 10^{-2}$ & Down \\
\hline MSTRG.16066.11 & -2.73949 & $6.82 \times 10^{-3}$ & Down \\
\hline NONMMUT031874.2 & -2.83355 & $3.91 \times 10^{-3}$ & Down \\
\hline NONMMUT058999.2 & -3.27548 & $4.24 \times 10^{-2}$ & Down \\
\hline NONMMUT149177.1 & -3.35448 & $2.38 \times 10^{-5}$ & Down \\
\hline NONMMUT068763.2 & -3.62327 & $1.03 \times 10^{-2}$ & Down \\
\hline NONMMUT031873.2 & -3.78681 & $1.71 \times 10^{-5}$ & Down \\
\hline ENSMUST00000181265 & -4.15283 & $4.42 \times 10^{-3}$ & Down \\
\hline NONMMUT142728.1 & -4.85759 & $3.13 \times 10^{-2}$ & Down \\
\hline NONMMUT001350.2 & -4.89458 & $1.59 \times 10^{-2}$ & Down \\
\hline NONMMUT051901.2 & -4.94198 & $1.18 \times 10^{-2}$ & Down \\
\hline
\end{tabular}

Down lncRNAs showing up or downregulated expression levels in the HFD + RSV vs. HFD group. lncRNA, long non-coding RNA; HFD, high-fat diet; RSV, resveratrol.

and 96 differentially expressed mRNAs (30 upregulated and 66 downregulated) were determined (Tables II and III). The distribution and trend of differential expression levels of lncRNAs and mRNAs was observed using a heat map (Fig. 1). The scatter plot displays the distribution and approximate number of lncRNAs and mRNAs showing $>2$-fold-change in expression levels (Fig. 2A).
$R T-q P C R$ to verify the expression levels of miRNAs. A total of four downregulated lncRNAs (NONMMUT119418.1, NONMMUT058999.2, NONMMUT068763.2 and NONMMUT051901.2) and four upregulated lncRNAs (NONMMUT010559.5.2, NONMMUT059852.2, NONMMUT027048.2 and NONMMUT001352.2) randomly selected from the 58 lncRNAs showing differential expression 
Table III. Expression level patterns of mRNAs in HFD + RSV vs. HFD mice.

\begin{tabular}{|c|c|c|c|}
\hline Gene name & $\log _{2}$, fold-change & Q-value & Regulation, HFD + RSV vs. HFD \\
\hline Rnu3b4 & 6.33208 & $3.88 \times 10^{-9}$ & Up \\
\hline $\operatorname{mir} 8114$ & 6.22832 & $3.19 \times 10^{-2}$ & Up \\
\hline Gm27640 & 5.12685 & $2.59 \times 10^{-16}$ & Up \\
\hline Gm45753 & 4.84454 & $2.92 \times 10^{-9}$ & Up \\
\hline $\mathrm{Gm} 28373$ & 4.68876 & $1.59 \times 10^{-2}$ & Up \\
\hline Capn11 & 4.46449 & $1.51 \times 10^{-3}$ & Up \\
\hline Usf3 & 2.97090 & $1.67 \times 10^{-10}$ & Up \\
\hline Gm20427 & 2.66816 & $1.06 \times 10^{-2}$ & Up \\
\hline Gm38283 & 2.37695 & $1.23 \times 10^{-2}$ & Up \\
\hline Gm45301 & 2.28532 & $5.97 \times 10^{-3}$ & Up \\
\hline Igfbp1 & 2.20251 & $2.31 \times 10^{-3}$ & Up \\
\hline 4933431G14Rik & 2.15402 & $3.09 \times 10^{-2}$ & Up \\
\hline Pitx3 & 2.07722 & $4.26 \times 10^{-2}$ & Up \\
\hline Gm43314 & 1.96046 & $5.51 \times 10^{-6}$ & Up \\
\hline Gm38357 & 1.90174 & $6.14 \times 10^{-3}$ & Up \\
\hline Cyp2b10 & 1.88210 & $4.76 \times 10^{-6}$ & Up \\
\hline Gm28323 & 1.79929 & $3.89 \times 10^{-5}$ & Up \\
\hline Gm15344 & 1.67885 & $1.31 \times 10^{-3}$ & Up \\
\hline Noct & 1.62225 & $7.22 \times 10^{-3}$ & Up \\
\hline Gm45792 & 1.61784 & $1.59 \times 10^{-2}$ & Up \\
\hline Gpc1 & -1.01448 & $8.54 \times 10^{-3}$ & Down \\
\hline Fdps & -1.03416 & $7.48 \times 10^{-3}$ & Down \\
\hline Rhbg & -1.08002 & $3.09 \times 10^{-2}$ & Down \\
\hline Calm1 & -1.11653 & $8.91 \times 10^{-4}$ & Down \\
\hline 2510016D11Rik & -1.16015 & $1.06 \times 10^{-2}$ & Down \\
\hline Gm5873 & -1.18954 & $1.59 \times 10^{-2}$ & Down \\
\hline Nrep & -1.18980 & $2.27 \times 10^{-3}$ & Down \\
\hline Mesd & -1.19613 & $8.22 \times 10^{-3}$ & Down \\
\hline Camk2b & -1.20313 & $1.73 \times 10^{-2}$ & Down \\
\hline Mki67 & -1.21546 & $6.09 \times 10^{-4}$ & Down \\
\hline Wfdc2 & -1.22930 & $7.48 \times 10^{-3}$ & Down \\
\hline Pcsk9 & -1.24132 & $3.49 \times 10^{-2}$ & Down \\
\hline Synj2 & -1.27207 & $1.41 \times 10^{-4}$ & Down \\
\hline Neurl1b & -1.27456 & $3.05 \times 10^{-2}$ & Down \\
\hline Gm3571 & -1.32982 & $3.77 \times 10^{-3}$ & Down \\
\hline $\operatorname{Rgs} 3$ & -1.34144 & $5.51 \times 10^{-6}$ & Down \\
\hline Cntnap1 & -1.39291 & $2.68 \times 10^{-2}$ & Down \\
\hline Socs 2 & -1.41122 & $7.95 \times 10^{-4}$ & Down \\
\hline Gm27702 & -1.41732 & $2.79 \times 10^{-2}$ & Down \\
\hline Phlda1 & -1.50249 & $2.39 \times 10^{-5}$ & Down \\
\hline
\end{tabular}

Down Top 20 mRNAs showing up or downregulated expression levels in HFD + RSV vs. HFD group. HFD, high-fat diet; RSV, resveratrol.

levels in the HFD + RSV and HFD groups following RSV intervention were evaluated using RT-qPCR to verify the sequencing results. The expression levels of these eight IncRNAs were consistent with those demonstrated by sequencing analysis of the HFD + RSV and HFD groups. Compared with the CON group, there was no significant difference in the expression levels of lncRNAs in the HFD group except for those of NONMMUT059852.2 and
NONMMUT027048.2 (Fig. 3). Although these two sets of verification results were inconsistent with the sequencing results, similar trends were obtained. The discrepancy may be related to the selection of samples and the size of the sample size.

The sequencing company produced only second-level results. The distribution of enriched differential genes at the three levels of GO is presented as a histogram, showing 
A

B
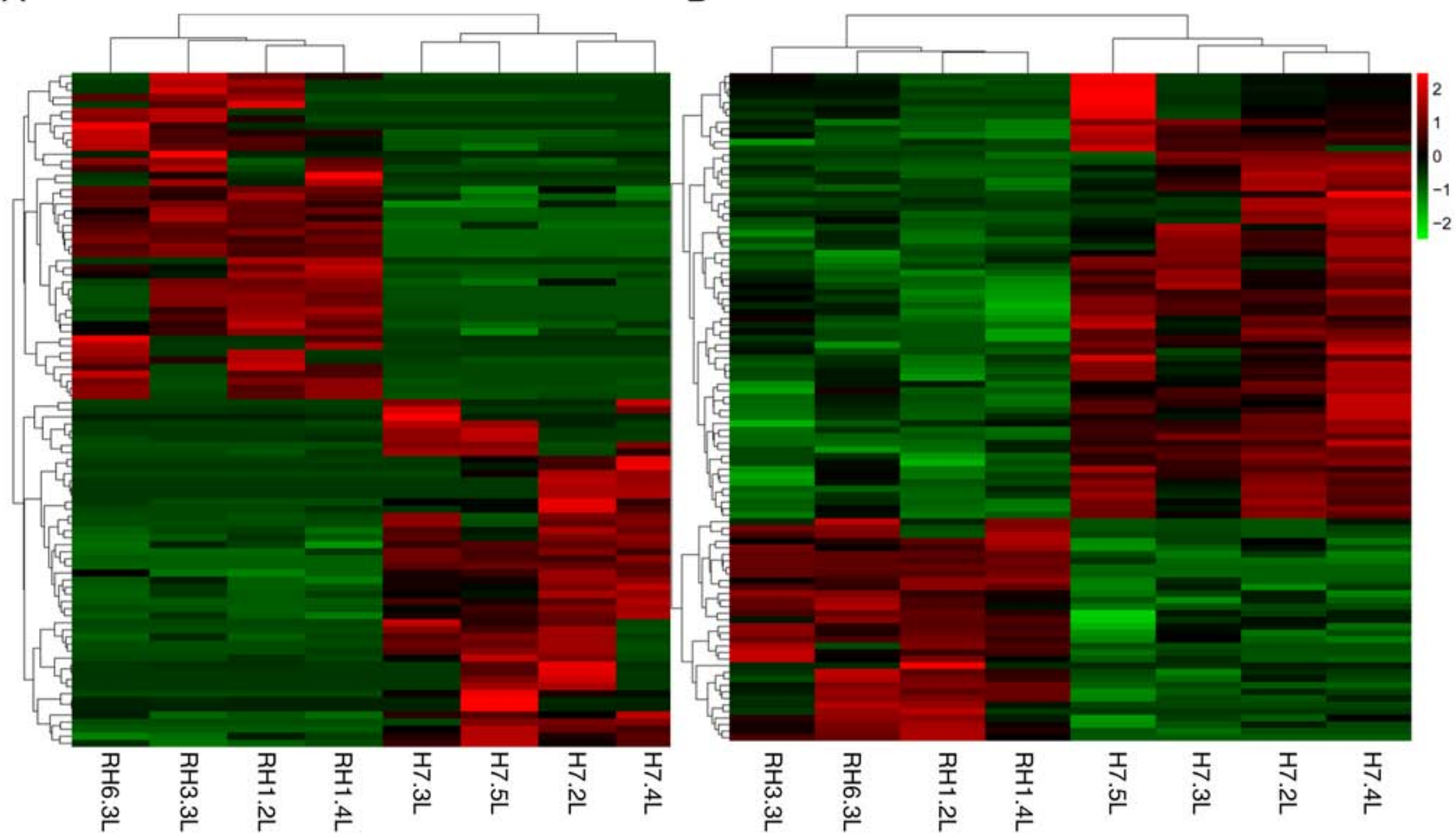

Figure 1. Clustering heat map of lncRNAs and mRNAs variation between HFD + resveratrol and HFD. (A) lncRNAs. (B) mRNAs. Red, fold-change $\geq 2$. Green, fold-change $\leq 2$. P $<0.05$. lncRNA, long non-coding RNA; HFD, high-fat diet.

A

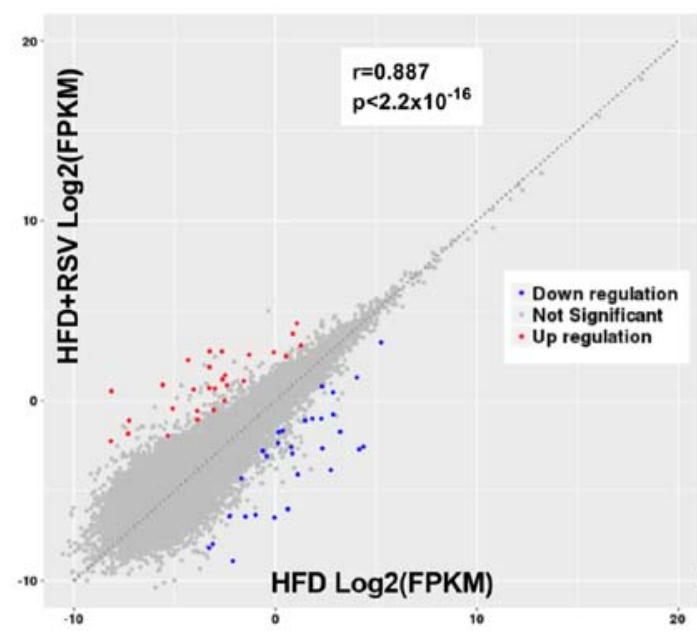

B

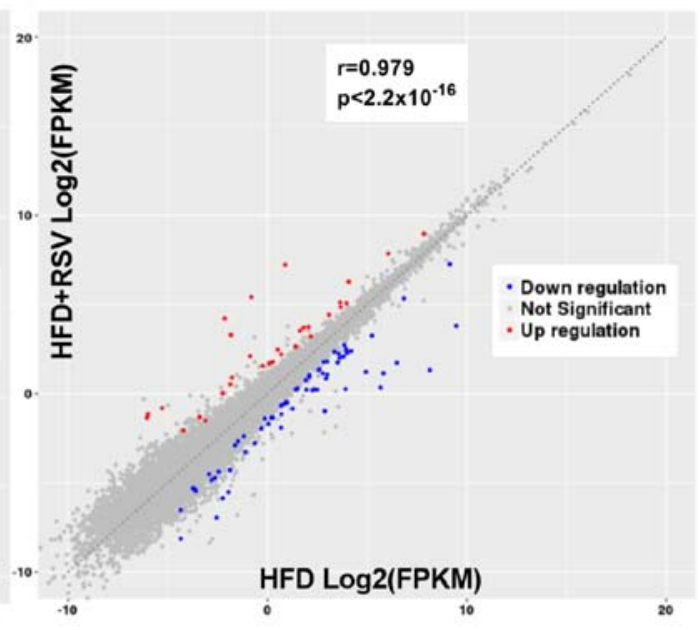

Figure 2. Scatter plot of variations in lncRNAs and mRNAs in HFD + RSV vs. HFD. (A) lncRNAs. (B) mRNAs. Red, fold-change $\geq 2$; Blue, fold-change $\leq 2$. $\mathrm{P}<0.05$. IncRNA, long non-coding RNA; HFD, high-fat diet; RSV, resveratrol; FPKM, fragments per kilobase of million mapped reads.

the second-level results in the GO database. The significant BPs in the GO classification included the 'cellular process', 'single-organism process' and 'biological regulation'. CC primarily included the 'cell', 'organizer' and 'membrane'. MF included 'binding', 'catalytic activity' and 'molecular function regulator' (Fig. 4A). The differential gene-associated classes of the KEGG database included cellular processes, environmental information processing, genetic information processing, metabolism and organizational systems (Fig. 4B).

Enrichment analysis of $G O$ and $K E G G$. The significant BPs in the top $30 \mathrm{GO}$ enrichment analysis of mRNAs included 'steroid metabolic process', 'response to insulin', 'cellular response to insulin stimulus'. CC included the 'spindle', and MF included 'protein kinase regulator activity' and 'kinase regulator 
A

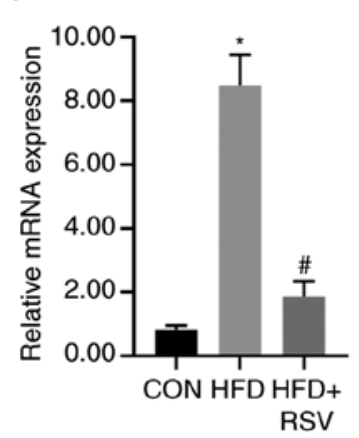

$\mathrm{E}$

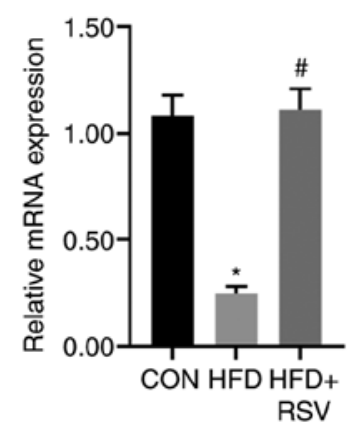

B

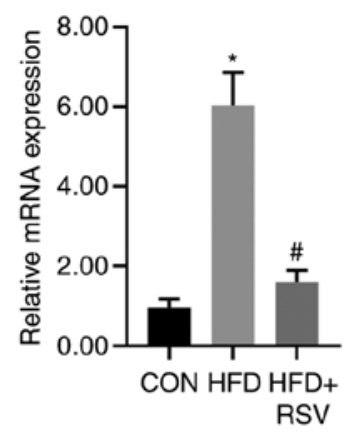

$\mathrm{F}$

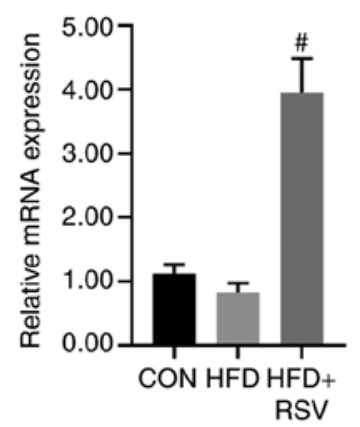

NONMMUT068763.2

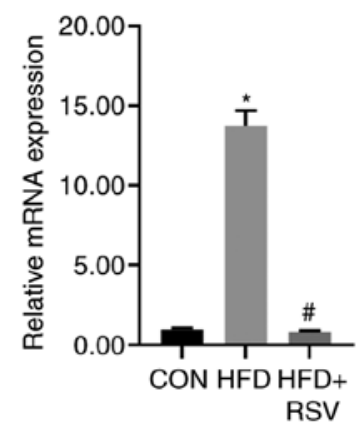

G

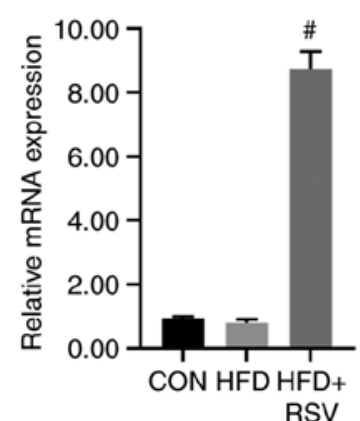

NONMMUT051901.2
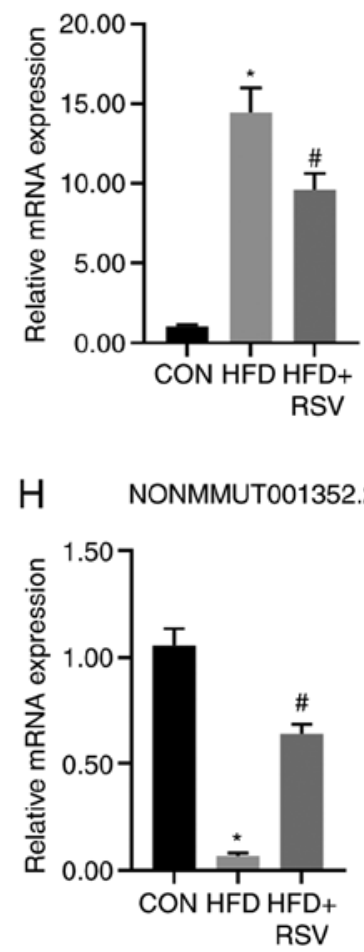

Figure 3. Validation of four upregulated and four downregulated lncRNAs by RT-qPCR in the HFD + RSV group compared to in the HFD group. (A) NONMMUT119418.1; (B) NONMMUT058999.2; (C) NONMMUT068763.2; (D) NONMMUT051901.2; (E) NONMMUT010559.2; (F) NONMMUT059852.2; (G) NONMMUT027048.2.; and (H) NONMMUT001352.2. All results were obtained from three independent experiments. Data are presented as the mean \pm standard deviation $(\mathrm{n}=4)$. One-way analysis of variance was used for statistical analysis followed by a post hoc least significant difference test (A-D F and G) or Tamhane's multiple comparison test ( $\mathrm{E}$ and $\mathrm{H}){ }^{*} \mathrm{P}<0.05$ vs. CON, ${ }^{\prime} \mathrm{P}<0.05$ vs. HFD. IncRNA, long non-coding RNA; RT-q, reverse transcription-quantitative; HFD, high-fat diet; RSV, resveratrol; CON, control.

activity' (Fig. 5A). mRNA KEGG pathway analysis was performed on the signaling pathways involving differentially expressed miRNAs. The top 30 pathway enrichments included the 'insulin', 'glucagon', 'prolactin', 'Jak-STAT', 'insulin resistance' and 'neurotrophin signaling' pathways. Among these, the insulin signaling pathway showed the highest score and was associated with the animal model of insulin resistance (Fig. 5B). This pathway was selected and the associated target genes SOCS3, G6PC, FOXO1 and PEPCK were predicted by pathway analysis (21). Through sequencing it was also found that among the eight verified lncRNAs, NONMMUT058999.2 is associated with SOCS3 and NONMMUT051901.2 is associated with G6PC.

Comparison of mRNA levels of insulin signaling pathway-associated genes. Compared with the CON group, the mRNA levels of SOCS3, G6PC, FOXO1, and PEPCK in the HFD group were significantly increased. The mRNA levels of SOCS3, G6PC, FOXO1 and PEPCK in the HFD + RSV group were significantly lower than those in the HFD group (Fig. 6).

Comparison of protein expression levels of insulin signaling pathway-associated genes. Compared with the CON group, the expression levels of SOCS3, FOXO1, G6PC and PEPCK in the HFD group were significantly increased. Compared with the HFD group, the expression levels of SOCS3, FOXO1, G6PC and PEPCK were significantly decreased in the HFD + RSV group (Fig. 7).
Combined analysis of differentially expressed lncRNAs and $m R N A s$. The sections belonging to differential mRNAs in the target genes of differential lncRNAs were isolated and assessed for enrichment in the GO and KEGG databases. A total of nine lncRNAs and mRNAs were simultaneously differentially expressed (Table IV).

lncRNA-miRNA-mRNA co-expression network. By calculating the dynamic gene expression levels in the HFD + RSV and HFD groups and calculating the co-expression association between genes, the present study constructed a network map of associated lncRNAs, miRNAs and mRNAs. This demonstrated that the lncRNAs NONMMUT147434.1 and NONMMUT145297.1 regulate the majority of miRNAs in this network. NONMMUT147434.1 regulates miRNA mmu-miR-1195, mmu-miR-3104-5p, mmu-miR-709, mmu-miR-7667-5p and mmu-miR-574-5p. NONMMUT145297.1 regulates mmu-miR-3473b, mmu-miR-3473e, mmu-miR-7032-5p, mmu-miR-328-5p and mmu-miR-466i-5p (Fig. 8).

Effect of RSV on insulin signaling pathway following knockdown of NONMMUT058999.2. From the eight selected lncRNAs, NONMMUT058999.2 exhibited the highest FPKM value. The pathway analysis revealed that SOCS3 was associated with NONMMUT058999.2, presenting a consistent trend. Following knockdown of NONMMUT058999.2, the expression levels of SOCS3, FOXO1, G6PC and PEPCK were significantly higher in PA compared with the CON 


\section{A GO classification}

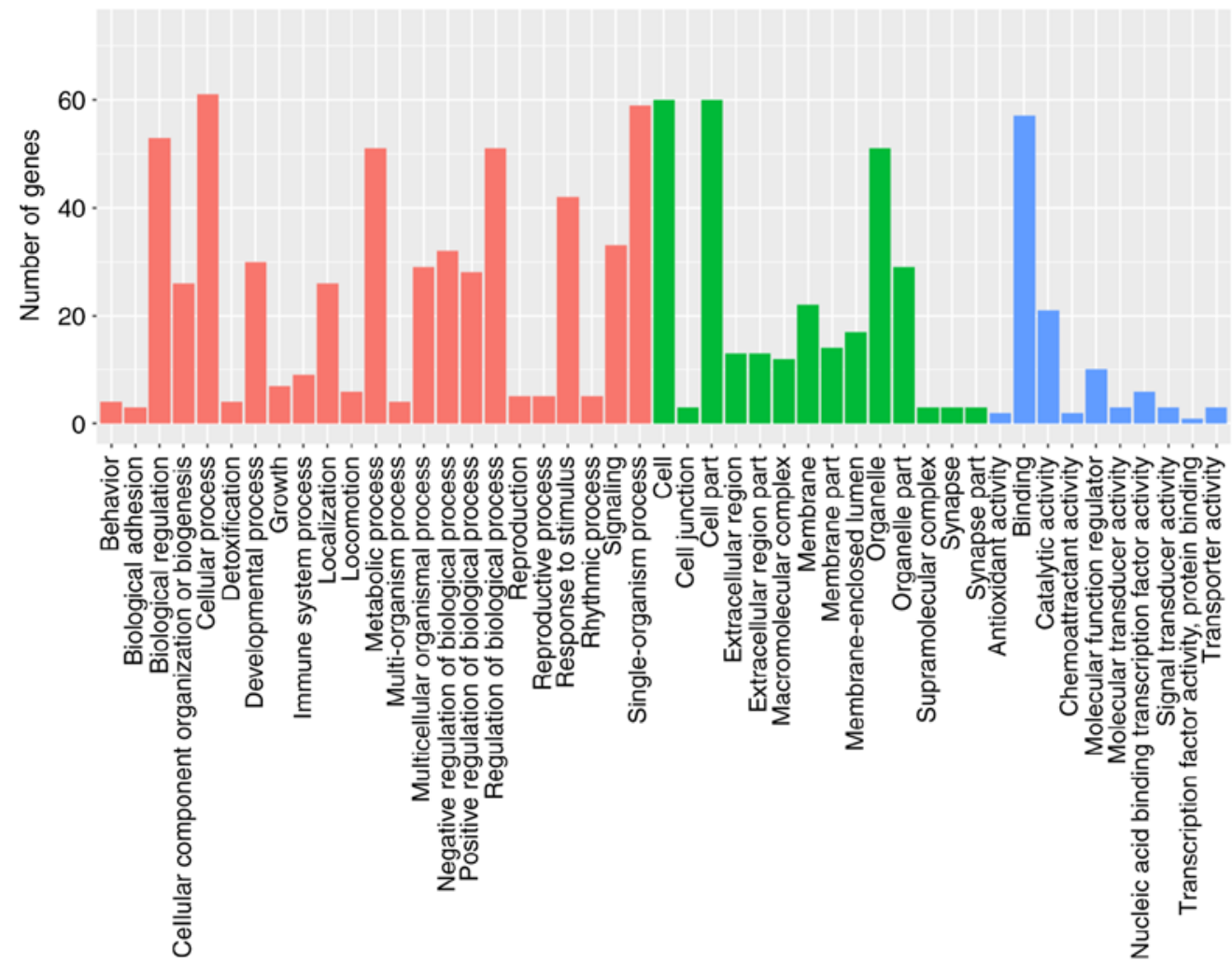

GO_domain

Biological_process

Cellular_component

Molecular_function
B

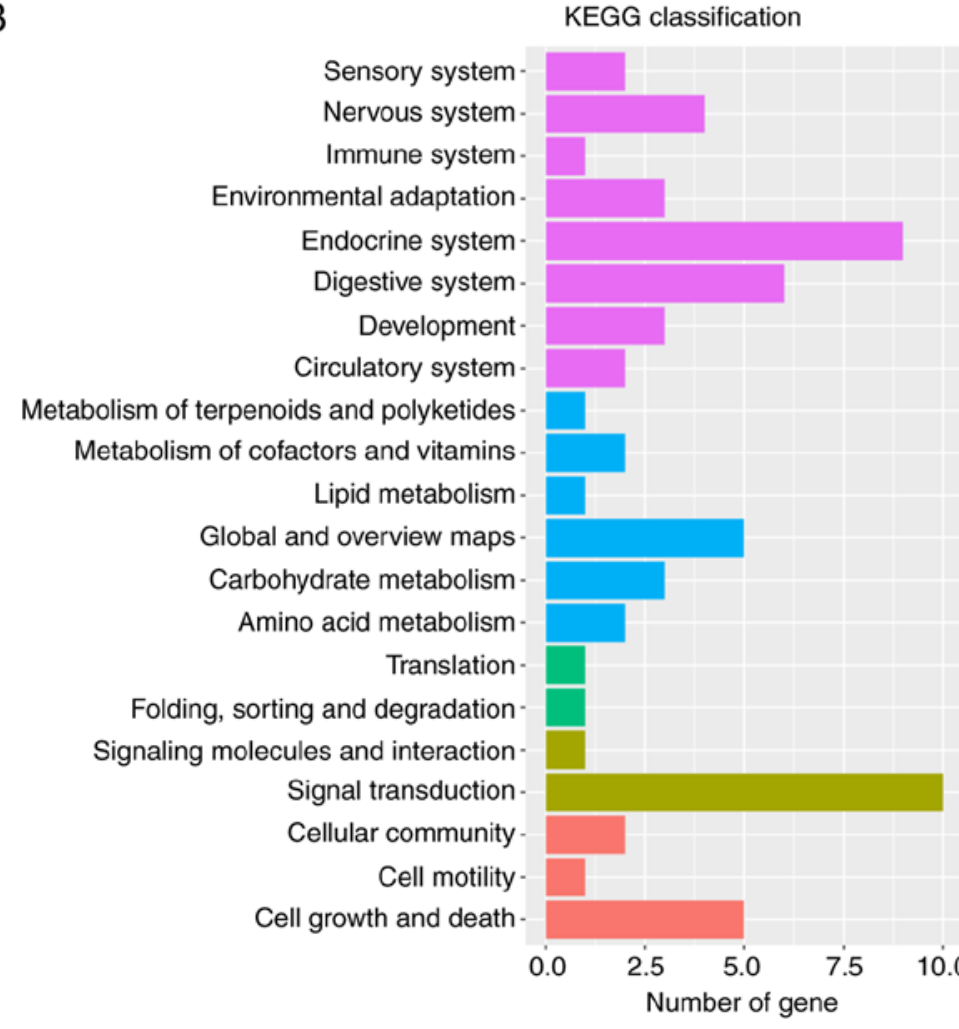

\section{Class}

Cellular processes

Environmental information processing

Genetic information processing

Metabolism

Organismal systems

Figure 4. GO and KEGG function distribution of differentially expressed mRNAs. (A) GO classification analysis of differentially expressed mRNAs. (B) KEGG classification analysis of differentially expressed mRNAs. GO, Gene Ontology; KEGG, Kyoto Encyclopedia of Genes and Genomics.

group. Furthermore, knockdown of NONMMUT058999.2 significantly decreased the expression levels of SOCS3, FOXO1, G6PC and PEPCK. The expression levels of SOCS3, FOXO1, G6PC and PEPCK were significantly lower in the
PA + RSV than in the PA group. Compared with knockdown of NONMMUT058999.2, no significant changes in the expression levels were observed for SOCS3, FOXO1, G6PC, and PEPCK in the PA + RSV group (Fig. 9). 
A

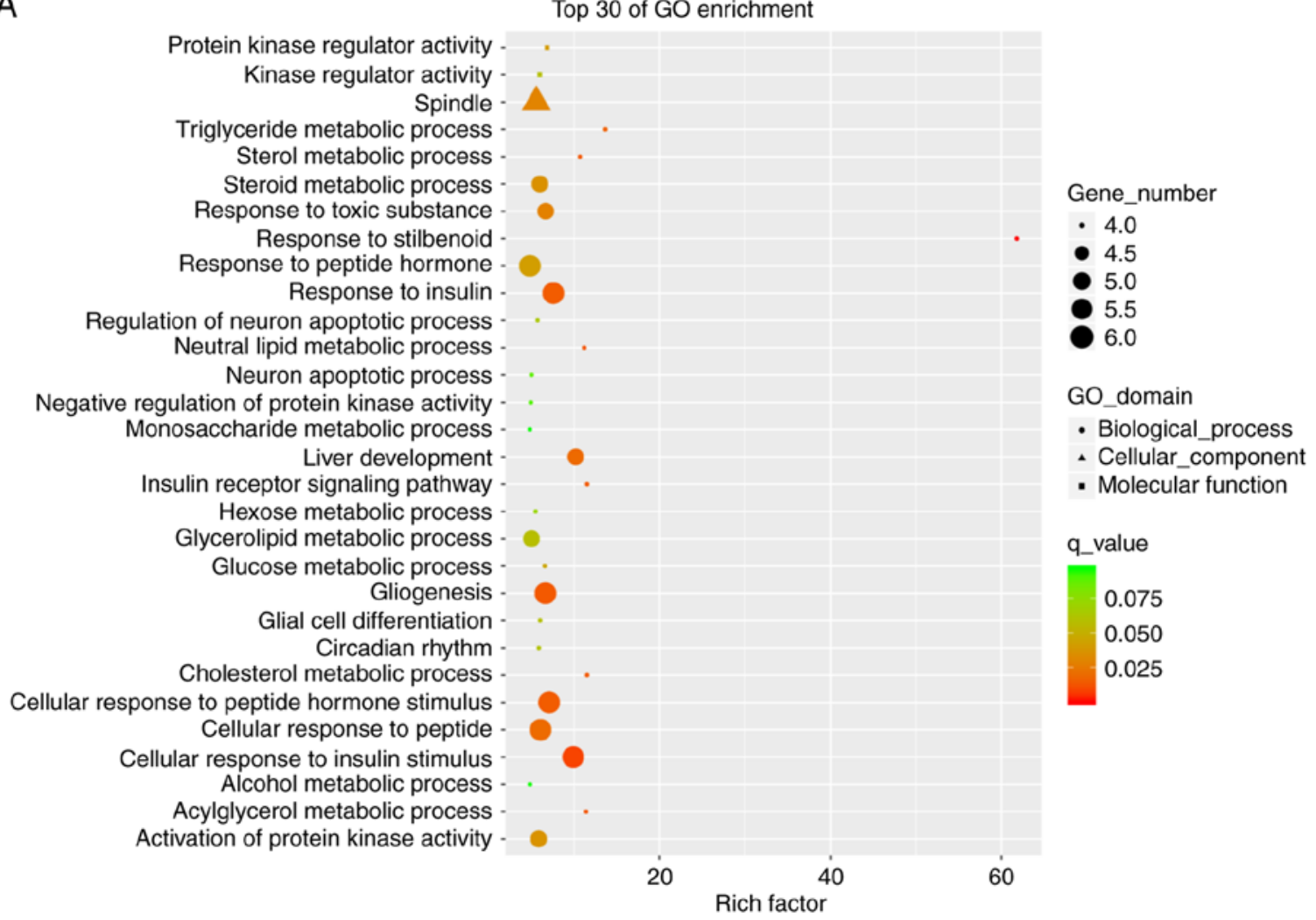

B

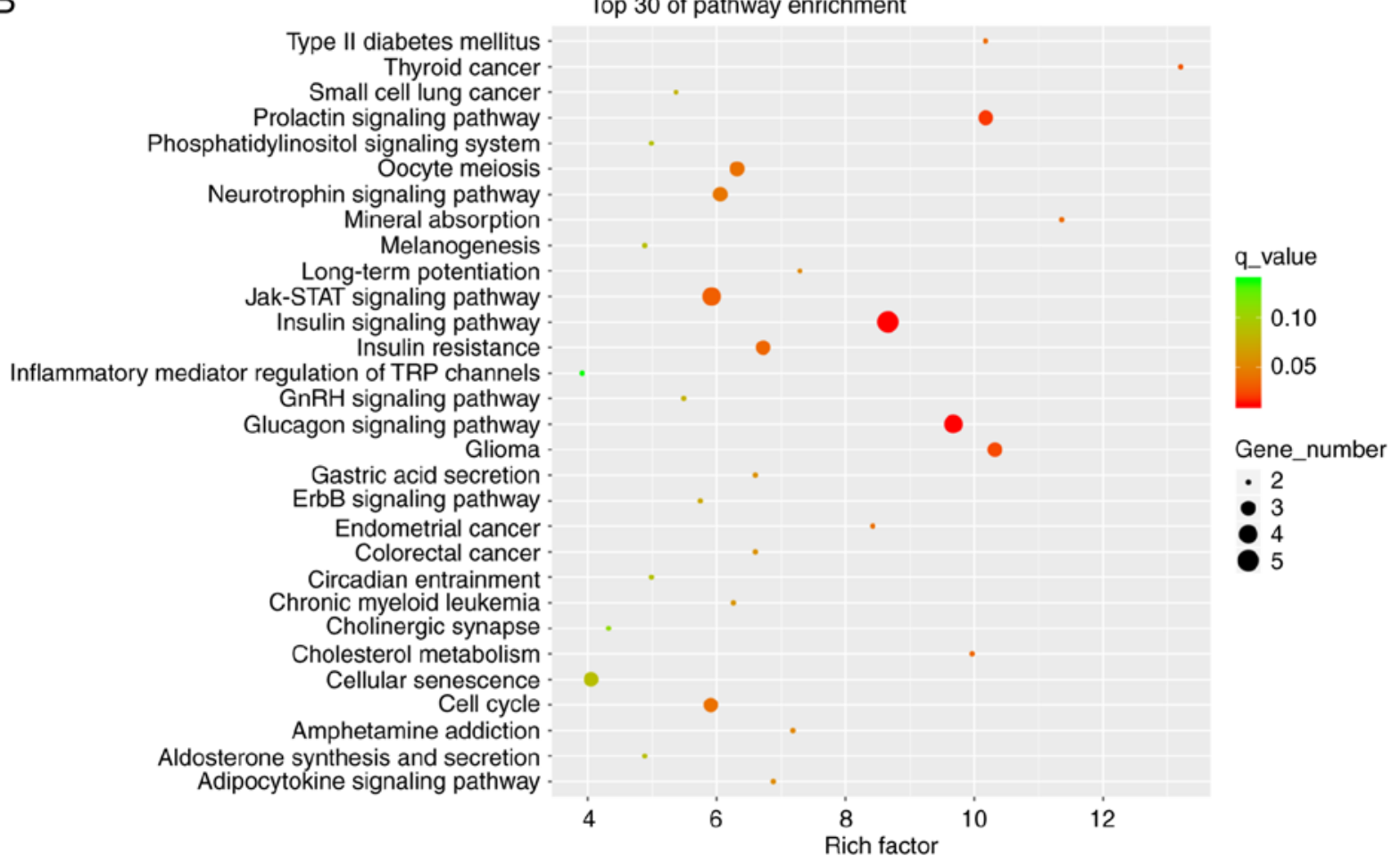

Figure 5. GO and pathway analysis of differentially expressed mRNAs. (A) GO analysis of differentially expressed mRNAs. (B) KEGG pathway analysis of differentially expressed mRNAs. GO, Gene Ontology; KEGG, Kyoto Encyclopedia of Genes and Genomics.

\section{Discussion}

RSV, a phytoalexin of polyphenols, has potential for improving insulin resistance and treating metabolic diseases such as diabetes (22). A previous study demonstrated that
RSV can activate insulin signaling pathways, increase insulin sensitivity, protect pancreatic islet $\beta$ cells, decrease lipid production, stimulate fatty acid oxidation and regulate intestinal flora (23). The liver is the primary site of glucose and lipid metabolism and is sensitive to insulin. Metabolic 
A

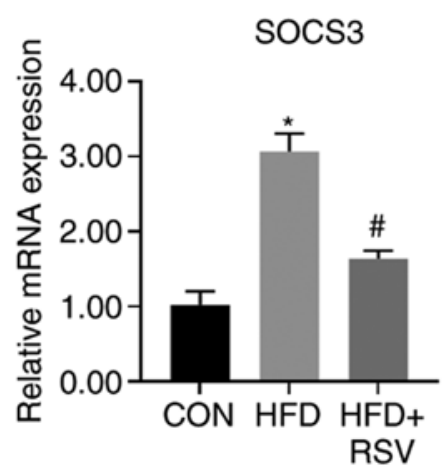

C

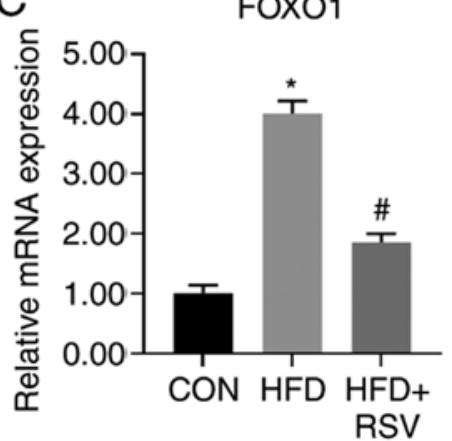

B

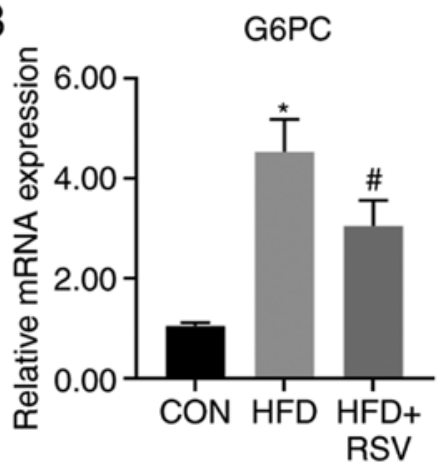

D

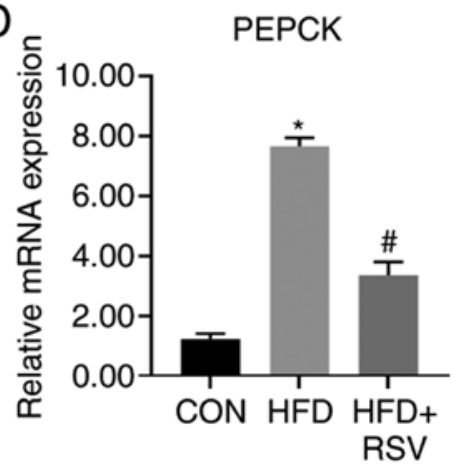

Figure 6. Relative mRNA expression of insulin signal pathway indicators in mouse liver. (A) SOCS3; (B) G6PC; (C) FOXO1; (D) PEPCK. Data are presented as the mean \pm standard deviation $(n=4)$. One-way analysis of variance was used for statistical analysis followed by a post hoc least significant difference test. ${ }^{*} \mathrm{P}<0.05$ vs. CON, ${ }^{\#} \mathrm{P}<0.05$ vs. HFD. CON, control; HFD, high-fat diet.

A

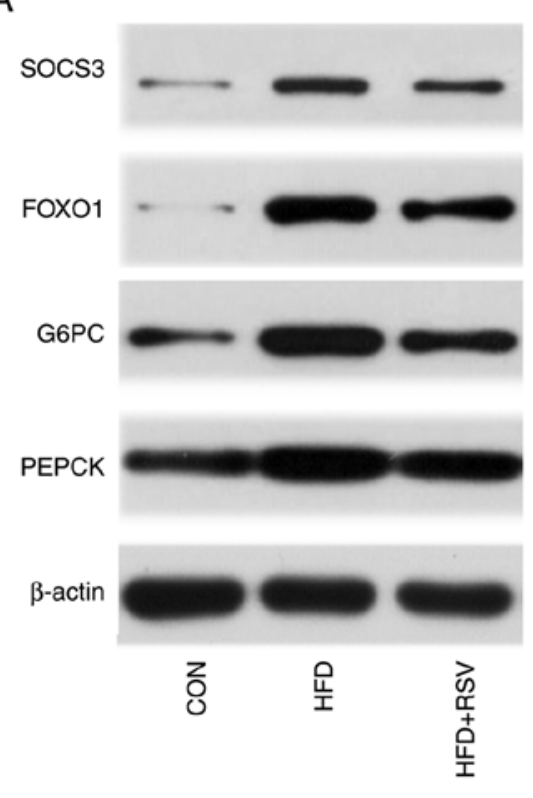

B

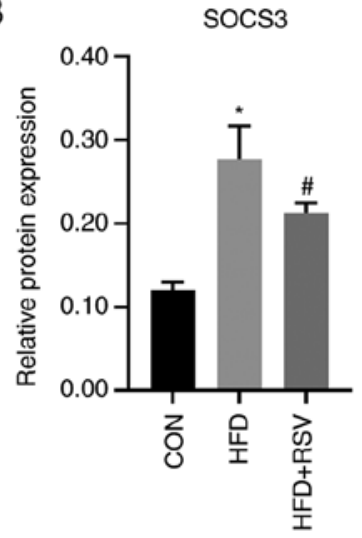

D

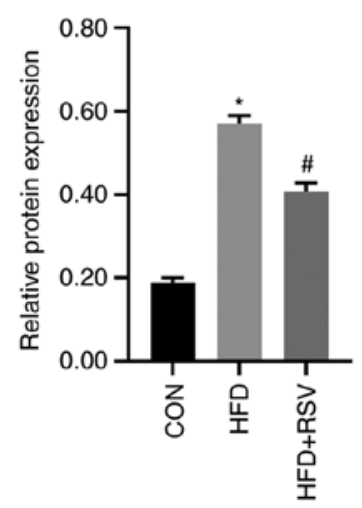

C

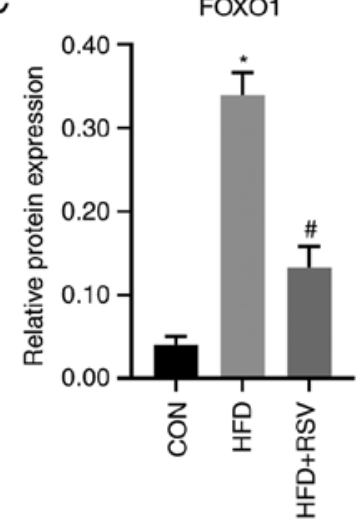

E

E PEPCK

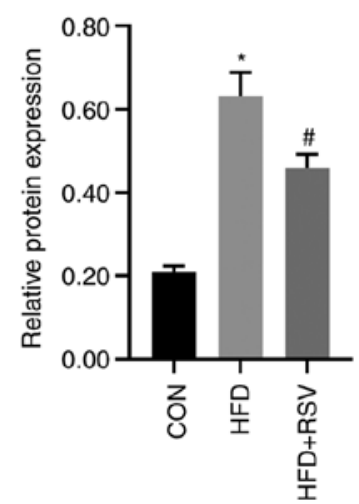

Figure 7. Relative protein expression of insulin signaling pathway indicators in mice liver. (A) Protein bands of insulin signal pathway molecules; (B) SOCS3; (C) FOXO1; (D) G6PC; (E) PEPCK. Densitometric analysis of protein expression. Data are presented as the mean \pm standard deviation (n=3). One-way ANOVA was used for statistical analysis followed by a post hoc least significant difference test. "P<0.05 vs. CON, ${ }^{*} \mathrm{P}<0.05$ vs. HFD. CON, control; HFD, high-fat diet. 
Table IV. Combined analysis of differential lncRNAs and mRNAs in HFD + RSV vs. HFD mice.

\begin{tabular}{lccccc}
\hline Gene & $\log _{2}$ FC-lncRNAs & Regulation & Gene name & $\log _{2}$ FC-mRNAs & Regulation \\
\hline NONMMUT119418.1 & -2.578527568 & Down & Aacs & -1.812654567 & Down \\
NONMMUT031874.2 & -2.833546204 & Down & Nrep & -1.189799355 & Down \\
NONMMUT031873.2 & -3.786814191 & Down & Nrep & -1.189799355 & Down \\
ENSMUST00000156612 & -2.001634041 & Down & Apoa4 & -1.881578742 & Down \\
MSTRG.5260.1 & -2.402628858 & Down & Camk2b & -1.203129759 & Down \\
NONMMUT144314.1 & -6.791820344 & Down & Knl1 & -1.569105399 & Down \\
NONMMUT059480.2 & -2.636807826 & Down & Bhlhe41 & -1.54587736 & Down \\
ENSMUST00000181265 & -4.152826048 & Down & Dlgap5 & -2.180235362 & Down \\
NONMMUT149177.1 & -3.354476528 & Down & Aacs & -1.812654567 & Down \\
\hline
\end{tabular}

Combined analysis of differential lncRNAs and mRNA expression levels in HFD + RSV vs. HFD group. IncRNA, long non-coding RNA; HFD, high-fat diet; RSV, resveratrol.

disorders associated with the liver can cause numerous metabolic syndromes, the most common of which are obesity, diabetes and non-alcoholic fatty liver disease (24). In type 2 diabetes, excessive gluconeogenesis increases endogenous processes in grape skin production, elevates blood sugar levels and induces IR (25). The gluconeogenesis pathway is regulated by two rate-limiting enzymes, G6PC and PEPCK, which are regulated by FOXO1 (26). FOXO1 is a key molecule in the insulin signaling pathway and is regulated by PI3K-AKT. Phosphorylated PI3K activates AKT, which affects downstream pathways. AKT phosphorylation inhibits FOXO1 transcription, decreasing its expression level and further inhibiting G6PC and PEPCK, thus lowering blood glucose levels and improving IR (27). SOCS3 is a negative feedback regulator of the cytokine activation pathway (28). A previous study showed that IR in diabetic mice is significantly improved following SOCS3 knockdown (29). The expression level of SOCS3 in an insulin-resistant obese mice model was higher than that in a normal group (30). The expression level of SOCS3 is higher in patients with type 2 diabetes than that in the normal population (31). These studies suggest that SOCS3 is associated with IR (32). SOCS3 can decrease phosphorylation or degrade protein expression levels by competitively binding insulin receptor substrate 1 (IRSs-1), decreasing the binding of IRSs-1 to PI3K, thus preventing PI3K-Akt-FOXO1 signaling. The insulin signaling pathway increases the expression levels of G6PC and PEPCK, which elevates blood glucose and induces IR (33).

Previous studies have shown that lncRNA can adsorb miRNAs by sponge adsorption. This forms competitive endogenous RNA and affects the function of associated target genes, indirectly regulating gene expression levels (34). In this way, lncRNAs are involved in regulating epigenetics, and play a key role in human disease (35). In type 2 diabetes, 55 lncRNAs were found to be differentially expressed in the blood of patients with type 2 diabetes and normal healthy people, with the three most significant differences associated with glycated hemoglobin (36). A number of lncRNAs are associated with IR and glucose levels in the peripheral blood of patients with type 2 diabetes (36). However, to the best of our knowledge, there have been few previous studies that have demonstrated that lncRNAs in the hepatic insulin signaling pathway are affected by RSV. The present study demonstrated the regulation of IncRNAs in the insulin signaling pathway in a mouse model of IR induced by HFD and RSV following HFD.

As demonstrated by high-throughput sequencing, there were 58 differentially expressed lncRNAs and 96 differentially expressed mRNAs in the HFD + RSV group compared with the HFD group. Studies have shown that RSV can inhibit gluconeogenesis and improve IR $(37,38)$. A total of eight lncRNAs were selected to verify that the RT-qPCR results were consistent with the sequencing results, which showed that RSV altered the expression levels of liver lncRNAs to improve IR. GO and KEGG enrichment analysis of these 58 mRNAs reversed by RSV showed that the insulin signaling pathway was most significantly associated with these lncRNAs. The present study used pathway analysis to predict which target genes SOCS3, G6PC, FOXO1 and PEPCK, are associated with the insulin signaling pathway, which were then verified using RT-qPCR and western blotting. Notably, lncRNA NONMMUT058999.2 is associated with mRNA SOCS3, lncRNA NONMMUT051901.2 and mRNA G6PC, which showed similar expression level patterns. This suggests that RSV plays a role in improving IR via NONMMUT058999.2 and NONMMUT051901.2, thus affecting the insulin signaling pathway. Following NONMMUT058999.2 knockdown, the present study analyzed changes in the insulin signaling pathway. RSV improved IR via regulating the expression levels of SOCS3, FOXO1, G6PC and PEPCK. Compared with knocked-down NONMMUT058999.2, no significant changes in the expression levels were observed for SOCS3, FOXO1, G6PC and PEPCK in PA+RSV. These results indicate that the pharmacological effects of RSV are similar to the downregulation of NONMMUT058999.2. RSV improves HFD-induced IR by regulating NONMMUT058999.2. Similarly, RSV can improve IR via other IncRNAs.

lncRNAs regulate mRNA expression levels via cis and trans interactions and competitive binding with miRNA (39). By combining differential lncRNAs with differential mRNAs, the present study found that HFD-induced downregulation of 


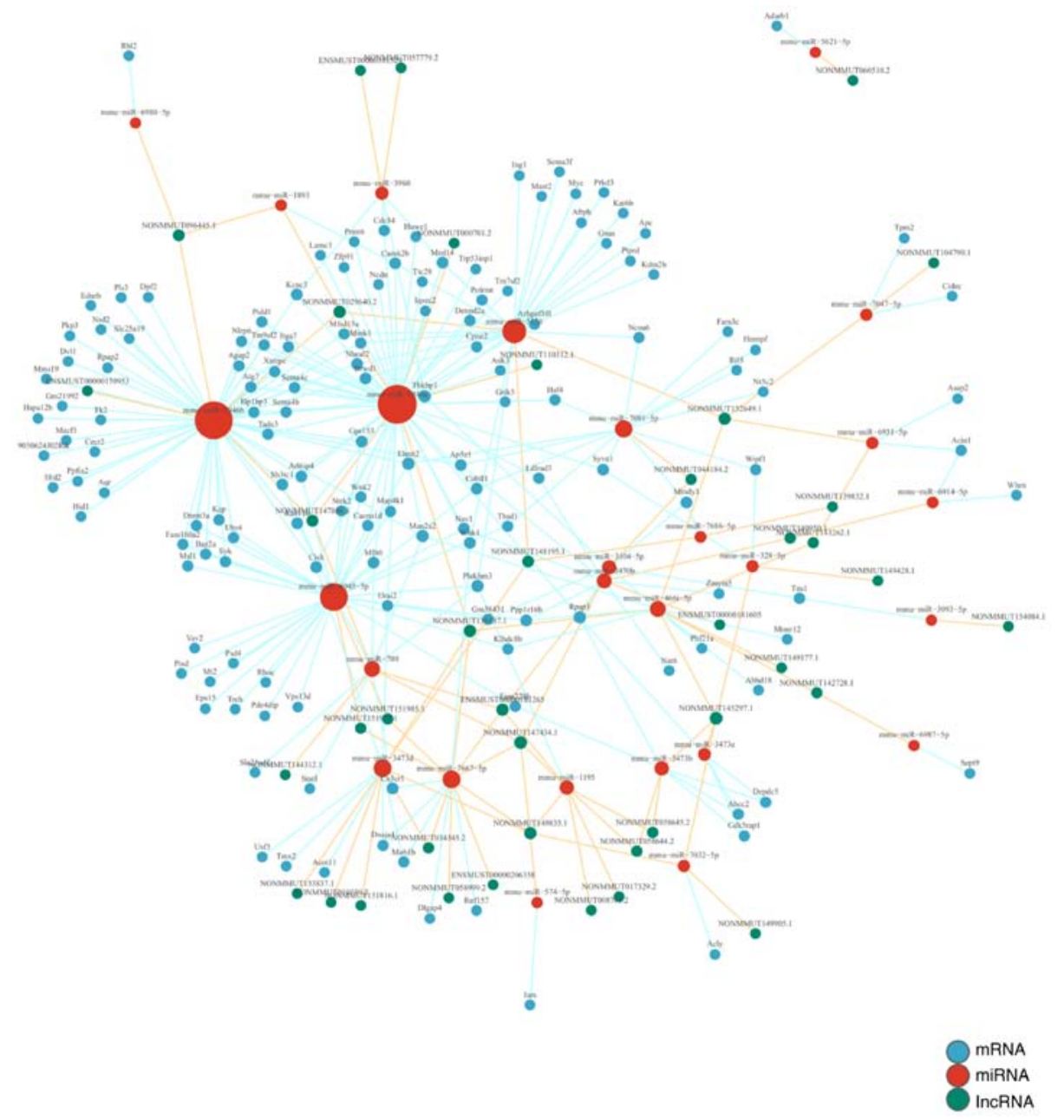

Figure 8. Top 400 differentially expressed lncRNA-miRNA-mRNA network. This co-expression network suggests inter-regulation of lncRNAs, miRNA, and mRNAs. lncRNA, long non-coding RNA; miRNA, microRNA.

A

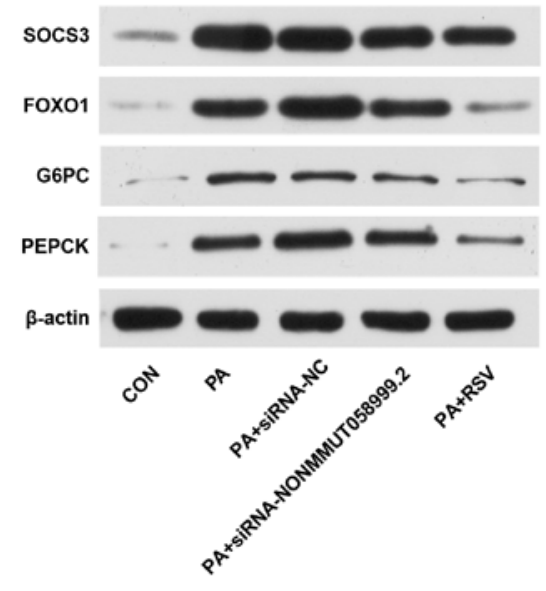

B

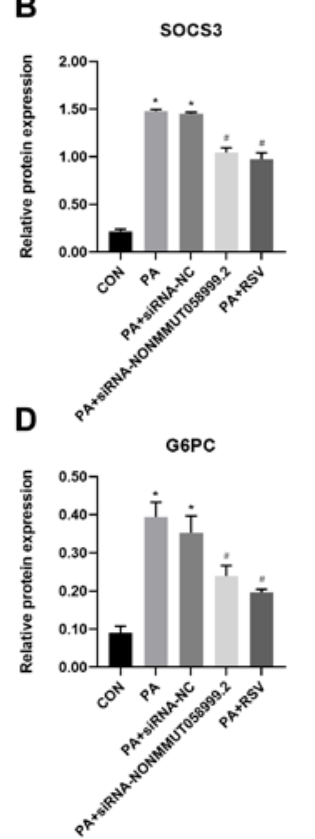

C

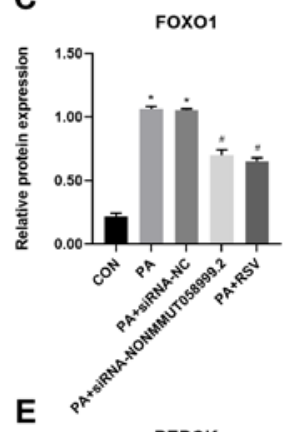

E PEPCK

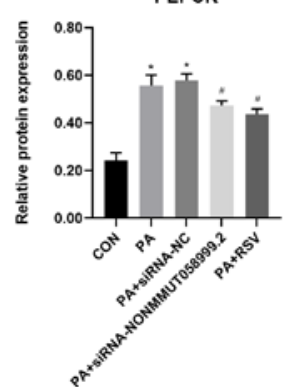

Figure 9. Effect of resveratrol on the insulin signaling pathway after knockdown of NONMMUT058999.2. (A) Protein bands of insulin signaling pathway molecules; (B) SOCS3; (C) FOXO1; (D) G6PC; and (E) PEPCK. Data are presented as the mean \pm standard deviation (n=3). One-way ANOVA was used for statistical analysis followed by a post hoc least significant difference test. ${ }^{*} \mathrm{P}<0.05$ vs. CON, ${ }^{\text {"P }}<0.05$ vs. PA. CON, control; PA, L-1 palmitic acid. 
nine lncRNAs and their corresponding mRNAs was reversed following RSV administration. Additionally, the co-expression network suggests inter-regulation of lncRNAs, miRNA and mRNAs. The present study demonstrated that two lncRNAs, NONMMUT147434.1 and NONMMUT145297.1, regulate the majority of miRNAs in this network. NONMMUT147434.1 regulates mmu-miR-1195, mmu-miR-3104-5p, mmu-miR-709, mmu-miR-7667-5p and mmu-miR-574-5p.NONMMUT145297.1 regulates mmu-miR-3473b, mmu-miR-3473e, mmu-miR-7032-5p, mmu-miR-328-5p and mmu-miR-466i-5p. A previous study showed that $\operatorname{lncRNAs}$ regulate mRNA expression levels via competitive binding with miRNA (40). These core lncRNAs may play a key role in improving IR (41). Although the exact mechanism of action of these lncRNAs remains unknown, the results of the present study provide a foundation for the development of novel diabetes treatments.

In conclusion, high-throughput sequencing revealed that IncRNAs were abnormally expressed following RSV intervention. These IncRNAs may be involved in the incidence and progression of type 2 diabetes. Further analysis suggested that lncRNAs play a role in the insulin signaling pathway, and that RSV may improve hepatic IR by regulating lncRNAs. The present study identified a novel biomarker or intervention target for RSV in the treatment of diabetes and contributes to understanding of the hypoglycemic mechanism of RSV. However, the functions and precise regulatory mechanisms of specific lncRNAs involved in improving IR require further investigation.

RSV can improve hepatic IR by regulating lncRNAs. RSV-regulated lncRNAs are potential therapeutic targets for type 2 diabetes.

\section{Acknowledgements}

Not applicable.

\section{Funding}

The present study was funded by the Natural Science Foundation of Hebei Province (grant no. H2018307071).

\section{Availability of data and materials}

The datasets used and/or analyzed during the current study are available from the corresponding author on reasonable request.

\section{Authors' contributions}

LS performed the experiments, analyzed the data and wrote the manuscript. GH performed the experiments and prepared the figures. HZ and WH established the mouse model. GS and HM designed the study and edited drafts of the manuscript. All authors contributed to data analysis, drafting or revising the article and agreed to be accountable for all aspects of the work. All authors read and approved the final manuscript.

\section{Ethics approval and consent to participate}

The experiment was supervised and approved by the Ethics Committee of the People's Hospital of Hebei Province (approval no. 201920) and performed in accordance with the Regulations on the Administration of Laboratory Animals.

\section{Patient consent for publication}

Not applicable.

\section{Competing interests}

The authors declare that they have no competing interests.

\section{References}

1. Ogurtsova K, da Rocha Fernandes JD, Huang Y, Linnenkamp U, Guariguata L, Cho NH, Cavan D, Shaw JE and Markaroff LE: IDF Diabetes Atlas: Global estimates for the prevalence of diabetes for 2015 and 2040. Diabetes Res Clin Pract 128: 40-50, 2017.

2. Akash MSH, Rehman K and Liaqat A: Tumor necrosis factor-alpha: Role in development of insulin resistance and pathogenesis of type 2 diabetes mellitus. J Cell Biochem 119: 105-110, 2018.

3. Rehman K, Akash MSH, Liaqat A, Kamal S, Qadir MI and Rasul A: Role of interleukin-6 in development of insulin resistance and type 2 diabetes mellitus. Crit Rev Eukaryot Gene Expr 27: 229-236, 2017.

4. Ku CR, Lee HJ, Kim SK, Lee EY, Lee MK and Lee EJ: Resveratrol prevents streptozotocin-induced diabetes by inhibiting the apoptosis of pancreatic $\beta$-cell and the cleavage of poly (ADP-ribose) polymerase. Endocr J 59: 103-109, 2012.

5. Li Q, Yue Y, Chen L, Xu C, Wang Y, Du L, Xue X, Liu Q, Wang Y and Fan F: Resveratrol sensitizes carfilzomib-induced apoptosis via promoting oxidative stress in multiple myeloma cells. Front Pharmacol 9: 334, 2018

6. Zhu X, Wu C, Qiu S, Yuan X and Li L: Effects of resveratrol on glucose control and insulin sensitivity in subjects with type 2 diabetes: Systematic review and meta-analysis. Nutr Metab (Lond) 14: 60, 2017.

7. Abbasi Oshaghi E, Goodarzi MT Higgins V and Adeli K: Role of resveratrol in the management of insulin resistance and related conditions: Mechanism of action. Crit Rev Clin Lab Sci 54: 267-293, 2017.

8. Zhao H, Shu L, Huang W, Song G and Ma H: Resveratrol affects hepatic gluconeogenesis via histone deacetylase 4. Diabetes Metab Syndr Obes 12: 401-411, 2019.

9. Hang Z, Yunjia Z, Linyi S, Guangyao S and Huijuan M: Resveratrol reduces liver endoplasmic reticulum stress and improves insulin sensitivity in vivo and in vitro. Drug Des Dev Ther 13: 1473-1485, 2019.

10. Wang X, Chang X, Zhang P, Fan L, Zhou T and Sun K: Aberrant expression of long non-coding RNAs in newly diagnosed type 2 diabetes indicates potential roles in chronic inflammation and insulin resistance. Cell Physiol Biochem 43: 2367-2378, 2017.

11. Gao Y, Wu F, Zhou J, Yan L, Jurczak MJ, Lee HY, Yang L, Mueller M, Zhou XB, Dandolo L, et al: The H19/let-7 double-negative feedback loop contributes to glucose metabolism in muscle cells. Nucleic Acids Res 42: 13799-13811, 2014.

12. Shu L, Zhao H, Huang W, Hou G, Song G and Ma H: Resveratrol Upregulates mmu-miR-363-3p via the PI3K-Akt pathway to improve insulin resistance induced by a high-fat diet in mice. Diabetes Metab Syndr Obes 13: 391-403, 2020.

13. Buchfink B, Xie C and Huson DH: Fast and sensitive protein alignment using DIAMOND. Nat Methods 12: 59-60, 2015.

14. Robinson MD, McCarthy DJ and Smyth GK: edgeR: A Bioconductor package for differential expression analysis of digital gene expression data. Bioinformatics 26, 139-140, 2010.

15. Zou Q, Mao Y, Hu L, Wu Y and Ji Z: miRClassify: An advanced web server for miRNA family classification and annotation. Comput Biol Med 45: 157-160, 2014.

16. Livak KJ and Schmittgen TD: Analysis of relative gene expression data using real-time quantitative PCR and the 2(-Delta Delta C(T)) method. Methods 25: 402-408, 2001.

17. Zhang H, Ge Z, Tang S, Meng R, Bi Y and Zhu D: Erythropoietin ameliorates PA-induced insulin resistance through the IRS/AKT/FOXO1 and GSK-3 $\beta$ signaling pathway, and inhibits the inflammatory response in HepG2 cells. Mol Med Rep 16: 2295-2301, 2017. 
18. Shu L, Hou G, Zhao H, Huang W, Song G and Ma H: Resveratrol improves high-fat diet-induced insulin resistance in mice by downregulating the lncRNA NONMMUT008655.2. Am J Transl Res 12: 1-18, 2020.

19. Li B and Dewey C: RSEM: Accurate transcript quantification from RNA-Seq data with or without a reference genome. BMC Bioinformatics 12: 323, 2011.

20. Grabherr MG, Haas BJ, Yassour M, Levin JZ, Thompson DA, Amit I, Adiconis X, Fan L, Raychowdhury R, Zeng Q, et al: Trinity: Reconstructing a full-length transcriptome without a genome from RNA-Seq data. Nat Biotechnol 29: 644-652, 2011.

21. Brasnyó P, Molnár GA, Mohás M, Markó L, Laczy B, Cseh J Mikolás E, Szijártó IA, Mérei A, Halmai R, et al: Resveratrol improves insulin sensitivity, reduces oxidative stress and activates the Akt pathway in type 2 diabetic patients. Br J Nutr 106: 383-389, 2011.

22. Thiel G and Rössler OG: Resveratrol regulates gene transcription via activation of stimulus-responsive transcription factors. Pharmacol Res 117: 166-176, 2017.

23. Rezaei Farimani A, Goodarzi MT, Saidijam M, Yadegarazari R, Zarei S and Asadi S: Effect of resveratrol on SNARE proteins expression and insulin resistance in skeletal muscle of diabetic rats. Iran J Basic Med Sci 22: 1408-1414, 2019.

24. Kim J, Bilder D and Neufeld TP: Mechanical stress regulates insulin sensitivity through integrin-dependent control of insulin receptor localization. Genes Dev 32: 156-164, 2018.

25. Hatting M, Tavares CDJ, Sharabi K, Rines AK and Puigserver P: Insulin regulation of gluconeogenesis. Ann N Y Acad Sci 1411: 21-35, 2018.

26. Langlet F, Haeusler RA, Lindén D, Ericson E, Norris T, Johansson A, Cook JR, Aizawa K, Wang L, Buettner C and Accili D: Selective inhibition of FOXO1 activator/repressor balance modulates hepatic glucose handling. Cell 171: 824-835. e18, 2017.

27. McCurdy CE, Schenk S, Holliday MJ, Philip A, Houck JA Patsouris D, MacLean PS, Majka SM, Klemm DJ and Friedman JE: Attenuated Pik3r1 expression prevents insulin resistance and adipose tissue macrophage accumulation in diet-induced obese mice. Diabetes 61: 2495-505, 2012.

28. Ma Z, Gong Y, Patel V, Karner CM, Fischer E, Hiesberger T, Carroll TJ, Pontoglio M and Igarashi P: Mutations of HNF-1beta inhibit epithelial morphogenesis through dysregulation of SOCS-3. Proc Natl Acad Sci USA 104: 20386-20391, 2007.

29. Galic S, Sachithanandan N, Kay TW and Steinberg GR Suppressor of cytokine signalling (SOCS) proteins as guardians of inflammatory responses critical for regulating insulin sensitivity. Biochem J 461: 177-188, 2014.

30. Fang S, Feng J, Zhang H, Li P, Zhang Y, Zeng Y, Cai Y, Lin X, Xue Y and Guan M: MiR-455 targeting SOCS3 improve liver lipid disorders in diabetic mice. Adipocyte 9: 179-188, 2020.
31. Pedroso JA, Buonfiglio DC, Cardinali LI, Furigo IC, Ramos-Lobo AM, Tirapegui J, Elias CF and Donato $\mathrm{J} \mathrm{Jr}$ : Inactivation of SOCS3 in leptin receptor-expressing cells protects mice from diet-induced insulin resistance but does not prevent obesity. Mol Metab 3: 608-618, 2014.

32. Abo El-Nasr NME, Saleh DO, Mahmoud SS, Nofal SM, Abdelsalam RM, Safar MM and El-Abhar HS: Olmesartan attenuates type 2 diabetes-associated liver injury: Cross-talk of AGE/RAGE/JNK, STAT3/SCOS3 and RAS signaling pathways Eur J Pharmacol 874: 173010, 2020.

33. Terán-Cabanillas E and Hernández J: Role of leptin and SOCS3 in inhibiting the type I interferon response during obesity. Inflammation 40: 58-67, 2017.

34. Qi X, Zhang DH, Wu N, Xiao JH, Wang X and Ma W: ceRNA in cancer: Possible functions and clinical implications. J Med Genet 52: 710-718, 2015.

35. Dong C, Liu S, Li Y and Cui Y: Serum lncRNA HAND2-AS1 is downregulated in diabetic patients with chronic renal failure and ameliorates cell apoptosis. Diabetol Metab Syndr 12: 39, 2020.

36. Sathishkumar C, Prabu P, Mohan V and Balasubramanyam M: Linking a role of lncRNAs (long non-coding RNAs) with insulin resistance, accelerated senescence, and inflammation in patients with type 2 diabetes. Hum Genomics 12: 41, 2018.

37. Zhao H, Chen S, Gao K, Zhou Z, Wang C, Shen Z, Guo Y, Li Z, Wan Z, Liu C and Mei X: Resveratrol protects against spinal cord injury by activating autophagy and inhibiting apoptosis mediated by the SIRT1/AMPK signaling pathway. Neuroscience 348: 241-251, 2017.

38. González-Rodríguez Á, Santamaría B, Mas-Gutierrez JA, Rada P, Fernández-Millán E, Pardo V, Álvarez C, Cuadrado A, Ros M, Serrano M and Valverde AM: Resveratrol treatment restores peripheral insulin sensitivity in diabetic mice in a sirt1-independent manner. Mol Nutr Food Res 59: 1431-1442, 2015.

39. Guttman M and Rinn JL: Modular regulatory principles of large non-coding RNAs. Nature 482: 339-346, 2012.

40. Ren XY, Han YD and Lin Q: Long non-coding RNA MIR155HG knockdown suppresses cell proliferation, migration and invasion in NSCLC by upregulating TP53INP1 directly targeted by miR-155-3p and miR-155-5p. Eur Rev Med Pharmacol Sci 24 4822-4835, 2020.

41. Chen K, Ma Y, Wu S, Zhuang Y, Liu X, Lv L and Zhang G: Construction and analysis of a lncRNA-miRNA-mRNA network based on competitive endogenous RNA reveals functional lncRNAs in diabetic cardiomyopathy. Mol Med Rep 20: 1393-1403, 2019.

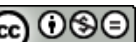

This work is licensed under a Creative Commons Attribution-NonCommercial-NoDerivatives 4.0 International (CC BY-NC-ND 4.0) License. 